\title{
Clinical Presentations of Pulmonary Embolism in the Emergency Department
}

\author{
Andreea Barcan, Dorin Tarta, Cristina Tarta \\ Clinic of Cardiology, Municipal Hospital, Mediaș, Romania
}

\section{ABSTRACT}

Pulmonary embolism (PE) is one of the most severe conditions encountered in the emergency department (ED) and one of the leading causes of cardiovascular morbidity and mortality, especially in patients presenting with hemodynamic instability, right ventricular dysfunction, or necessitating cardiopulmonary resuscitation. The early recognition and treatment of PE is essential, as many studies demonstrated that mortality rates drop significantly if adequate therapy is administered from the early stages. The aim of this update is to summarize the various patterns of PE presentations in the ED.

Keywords: pulmonary embolism, antiphospholipid syndrome, atrial fibrillation

\section{ARTICLE HISTORY}

Received: August 3, 2017

Accepted: September 20, 2017

\section{CORRESPONDENCE}

\section{Andreea Barcan}

Str. I.G. Duca nr. 33

551026 Mediaș, Romania

Tel: +40 269842550

E-mail: barcan_a@yahoo.com

\section{INTRODUCTION}

Pulmonary embolism (PE) is one of the leading cardiovascular causes of morbidity and mortality in patients surviving an acute coronary syndrome or stroke, and a large number of these patients die even before the diagnosis, due to the ambiguous forms of clinical presentation and lack of highly specific signs and symptoms. ${ }^{1,2}$ An important percent of these patients remains undiagnosed until the chronic phase of the disease, when pulmonary hypertension is installed, or in other cases, during autopsy. ${ }^{3,4}$ Large registry studies show that approximately $10 \%$ of $\mathrm{PE}$ patients die during the first three months after diagnosis. ${ }^{5,6}$ The early recognition and treatment of $\mathrm{PE}$ is essential, as many studies demonstrated that mortality rates drop significantly if adequate therapy is administered from the early stages. ${ }^{7}$

Echocardiography has become a cheap, helpful, and widely available method for the assessment of patients presenting with clinical suspicion for PE. Biomarkers such as D-dimers are useful for the exclusion of PE in low- and intermediate-risk patients, but current guidelines do not recommend it for the confirmation of the diagnosis. Computed tomography (CT) represents the method of choice for a positive diagnosis in intermediate and high pre-test probability risk patients. ${ }^{8,9}$

The clinical setting of PE patients is also an important factor for both short- and long-term survival. The 30 -day mortality rates vary from $1 \%$ to over $50 \%$, mainly depending on the baseline clinical characteristics of the patients. ${ }^{10}$ Those presenting in the emergency department (ED) with hemodynamic instability, right ventricular dysfunction, or necessitating cardiopulmonary resuscitation have mortality rates up to $70-80 \%$ in the acute phase. ${ }^{11}$ These parameters, along with altered left ventricular function, older age, higher body weight, and associated pulmonary and renal comorbidities, are also predictors 
for higher long-term mortality rates. ${ }^{11}$ The widespread use noninvasive diagnostic tools in the ED have led to better accuracy in the identification of even lower risk patients. The aim of this update is to summarize the various patterns of PE presentations in the ED.

\section{PE IN THE EMERGENCY DEPARTMENT}

The diagnosis of PE can be a very challenging task in the ED due to the diverse, common, and unspecific symptoms. The assessment of the large number of patients presenting with chest pain and dyspnea in the ED has to be accurate and cost-effective. McCaig et al. assert that approximately $15 \%$ of all ED patients would be screened for PE if every patient complaining of chest pain and shortness of breath would be tested. ${ }^{12}$ In severe cases, patients present to the ED with severe hypotension, tachyarrhythmia, or after an episode of cardiac arrest and cardiopulmonary resuscitation. All known risk factors, such as deep vein thrombosis, cancer, recent surgery, and immobilization, should be quickly evaluated in patients withsuspicion of $\mathrm{PE}$, as delayed diagnosis in the ED is associated with worse outcomes. ${ }^{13}$ The validated clinical probability scores and biomarkers help the ED physician to select the proper patient category who should undergo further investigations, as undiagnosed $\mathrm{PE}$ leads to high mortality rates, and unnecessary examinations create high costs and a possibly faulty diagnosis..$^{14-16}$

The triple-rule-out CT (TRO CT) is a new, emerging noninvasive diagnostic technique that is suitable to exclude the three most common, possibly life-threatening vascular conditions, namely acute coronary syndromes (ACS), aortic dissection, and PE, using decreased amount of radiation. It is an appropriate and cost-effective diagnostic tool for patients who present with chest pain in the ED and have low-to-intermediate risk for ACS. ${ }^{17-20}$ In a study which included 1,024 patients who presented to the ED for chest pain, Chae et al. demonstrated that TRO CT is a useful tool for an efficient triage of these patients and has a good predictive value for 30-day major adverse cardiovascular events (MACE), regardless of clinical scores. ${ }^{21}$ Further clinical studies are required for the validation of this method.

\section{CLINICAL PROBABILITY AND RISK STRATIFICATION}

Although the common symptoms and signs in PE are limited in sensitivity and specificity, clinical judgment, aided by the use of prediction scores, can provide a distribu- tion of patients with suspected PE into specific categories of probability. ${ }^{22}$ Through these scores, the algorithm sequence of predicting diagnosis will be based on binary decisions, thus inappropriate variance of clinical decision will be confined. ${ }^{22}$

The sequence of diagnostic work-up consist of applying pre-test probability rules, which are necessary in determining whether further invasive or noninvasive investigations are needed. There are several pre-test scores being used, either to rule-in or to rule-out PE diagnosis. The most applied validated pre-test scores are: the Geneva score with two derived models, the Wells score, the PISA models, the Charcotte model, the PESI scores, and the Gestalt model.

The original Geneva model (Wicki criteria) of risk stratification is based on assessment at the time of presentation. Similar to original Geneva score are the revised Geneva score and the simplified Geneva score. ${ }^{23}$ Nowadays, the revised Geneva Criteria are most used in determining PE probability risk. ${ }^{24}$ Patients presenting a total score equal or less than 2 points are considered unlikely to have PE. ${ }^{25}$

Another widely used probability risk score of PE is the Wells score, which was introduced in 1995 as a clinical prediction rule, then it was revised multiple times by modifying the cut-off value. It uses a cut-off value of 4, thus dividing patients into two categories, those with up to 4 points are unlikely to have PE, and those with more than 4 points are likely to have a current PE. ${ }^{26}$

A study conducted by El Wahsh et al., comparing different validated predicting scores of $\mathrm{PE}$, showed that among the most used scores the PISA model is the most accurate, capable of better negative prediction and better determination of positive prediction. ${ }^{27}$ According to the resulting percentage of probability, the patients' risk could be divided as follows: up to $10 \%$ low risk of $\mathrm{PE}$, between $10 \%$ and $50 \%$ intermediate risk of PE, $51 \%$ to $80 \%$ moderately high risk, and between $81 \%$ and $100 \%$ high risk of current PE. ${ }^{28}$

A meta-analysis comparing the 52 most important studies based on applying the most reliable risk scores in predicting PE, reveals that the Geneva score has the best sensitivity, while the revised Geneva model, the Wells score, and the Gestalt score have the most accurate specificity. These two important predicting scores of PE were analyzed as well in terms of failure rate, and the results showed that it is $0 \%$. The same meta-analysis compared the efficacy of each predicting score associated with assessment of $\mathrm{D}$-dimer value, and the results showed that the Wells score associated with $\mathrm{D}$-dimer value lacked in 
efficiency, while the Gestalt model associated with D-dimer was highly efficient. ${ }^{29}$

In order to assess the mortality and the adverse medical events in PE patients, the Pulmonary Embolism Severity Index (PESI) and the simplified PESI (sPESI) were introduced and validated..$^{30}$ The PESI stratifies patients in five risk classes corresponding to the 30-day mortality rate. The sPESI includes only 6 clinical factors, namely age over 80 years, malignancy, chronic cardiopulmonary disease, heart rate $>110 \mathrm{bpm}$, systolic blood pressure $<100 \mathrm{~mm} \mathrm{Hg}$, and $\mathrm{SaO} 2<90 \%$. If one of these parameters is present, a high risk of 30-day mortality and adverse events is associated. ${ }^{31}$ Current guidelines recommend the assessment of the PESI score at hospital admission and initiation of thrombolytic therapy. ${ }^{31}$

\section{SYNCOPE IN PE}

Until recently, syncope was considered an uncommon clinical presentation for $\mathrm{PE}$, and current guidelines and recommendations do not focus enough on the consideration and work-up of PE for these patients. However, in a recent, highly cited publication, Prandoni et al. identified a much higher prevalence $(17.3 \%)$ of PE in patients presenting in the ED for a first episode of syncope. ${ }^{32}$ Based on Prandoni's results, Oqab et al. performed a meta-analysis which included 6,608 patients from ED and found significantly lower prevalence of PE ( $\mathrm{p}<0.0001)$ in the same category of patients..$^{33}$ In a recent paper published by Frizell et al., the results of the Prandoni study are also contested, as only $1.4 \%$ of patients were identified with $\mathrm{PE}$ from a total number of 348 of patients presenting with syncope in the ED. ${ }^{34}$ These controverting results suggest the need of further investigations for the determination of the prevalence of $\mathrm{PE}$ in patients presenting with syncope in the ED.

\section{UNUSUAL PRESENTATIONS OF PE IN OUR EXPERIENCE}

Unusual presentations of PE can be frequently encountered in the ED. We report, for instance, the case of a patient with massive PE presenting to the emergency room for sudden onset of an atrial fibrillation (AF) episode. A 48-year-old male was admitted to our ED accusing recent onset dyspnea at mild exertion and palpitations. No significant family history was recorded. The patient suffered from deep vein thrombosis of the left inferior limb in the past, without other known diseases. The patient's blood pressure was $140 / 100 \mathrm{mmHg}$ with a heart rate of
$100 \mathrm{bpm}$, and no significant ECG changes were recorded. Cardiac ultrasound revealed a dilated right ventricle with an increased pulmonary pressure $(55 \mathrm{mmHg}$ ), with significant tricuspid regurgitation and an intracardiac hyperechogenic mobile mass of $13 / 10 \mathrm{~mm}$, localized in the right atrium and adherent to the intertribal septum, and a similar hyperechogenic mass in the right ventricle. A chest CT was performed, which showed bilateral massive pulmonary embolism. Considering the history of the patient and the multiple thrombotic events, aPL and anticardiolipin antibodies were determined and turned out to be positive, and the underlying cause of the PE proved to be antiphospholipid syndrome (APLS).

In order to investigate the cause of the thrombotic events which lead to $\mathrm{PE}$, it is important to consider other rare conditions in patients with unclear clinical presentation. Literature data shows that the acquired hypercoagulability status emphasized by a thrombotic event can be caused by APLS with an incidence of up to $2 \%$ in the general population and can consist in a hypercoagulability state with high implications in the cardiovascular system, with a 5-year mortality rate of 5.3\%.35-39 These patients are at high risk of developing arterial or venous thrombosis, intracardiac thrombus, pulmonary hypertension, coronary artery diseases, valvular disease, and stroke, and also can determine fetal loss..$^{40,41}$

The association between atrial fibrillation and PE is still highly debated and still understudied. Gex et al. suggest that $\mathrm{AF}$ increases the probability of $\mathrm{PE}$ in patients with chest pain and in a recent meta-analysis Bikdeli et al. state a strong relationship between $\mathrm{AF}$ and $\mathrm{PE}$, which needs further studies for elucidation..$^{42,43}$

\section{CONCLUSIONS}

$\mathrm{PE}$ can have various clinical presentations at the ED, starting from mild asymptomatic cases to more classical symptoms such as dyspnea and chest pain, or severe forms with malignant arrhythmias and cardiac arrest. It is also important to take into consideration atypical forms, such as atrial fibrillation or syncope, for an as low as possible rate of missed diagnosis and better short- and longterm outcomes. Clinical probability and risk stratification scores along with modern imagistic techniques help the ED physician for a quick and proper diagnosis of these patients.

\section{CONFLICT OF INTEREST}

Nothing to declare. 


\section{REFERENCES}

1. Heit JA, Silverstein MD, Mohr DN. The epidemiology of venous thromboembolism in the community. Thromb Haemost. 2001;86:452-463.

2. Jiménez Castro D, Sueiro A, Díaz G, et al. Prognostic significance of delays in diagnosis of pulmonary embolism. Thromb Res. 2007;121:153-158. doi: 10.1016/j.thromres.2007.03.028.

3. De Perrot $M$, Granton J, Fadel E. Pulmonary hypertension after pulmonary emboli: an underrecognized condition. CMAJ : Canadian Medical Association Journal. 2006;174:1706.

4. Kopcke D, Harryman O, Benbow EW, Hay C, Chalmers N. Mortality from pulmonary embolism is decreasing in hospital patients. Journal of the Royal Society of Medicine. 2011;104:327-331. doi: 10.1258/jrsm.2011.100395.

5. Aujesky D, Jimenez D, Mor MK, et al. Weekend versus weekday admission and mortality after acute pulmonary embolism. Circulation. 2009;119:962-968. doi: 10.1161/ CIRCULATIONAHA.108.824292.

6. Laporte S, Mismetti P, Decousus $\mathrm{H}$, et al. Clinical predictors for fatal pulmonary embolism in 15,520 patients with venous thromboembolism: findings from the Registro Informatizado de la Enfermedad TromboEmbolica venosa (RIETE) Registry. Circulation. 2008;117:1711-1716. doi: 10.1161/ CIRCULATIONAHA.107.726232.

7. Charlebois D. Early recognition of pulmonary embolism: the key to lowering mortality. J Cardiovasc Nurs. 2005;20:254259.

8. Konstantinides SV, Torbicki A, Agnelli G, et al. 2014 ESC guidelines on the diagnosis and management of acute pulmonary embolism. Eur Heart J. 2014;35:3033-3069, 3069a-3069k. doi: 10.1093/eurheartj/ehu479.

9. van Belle A, Buller HR, Huisman MV, et al. Effectiveness of managing suspected pulmonary embolism using an algorithm combining clinical probability, D-dimer testing, and computed tomography. JAMA. 2006;295:172-179. doi: 10.1001/ jama.295.2.172.

10. Janata K, Holzer M, Domanovits $\mathrm{H}$, et al. Mortality of patients with pulmonary embolism. Wien Klin Wochenschr. 2002;114:766-772.

11. Opincariu D, Mester A, Rațiu M, et al. Factors associated with one-year mortality in patients with acute pulmonary embolism. Journal of Cardiovascular Emergencies. 2016;2:6370. doi: 10.1515/jce-2016-0010.

12. McCaig LF, Burt CW. National hospital ambulatory medical care survey: 2002 emergency department summary. Adv Data. 2004;340:1-34.

13. Roy PM, Meyer G, Vielle B, et al. Appropriateness of diagnostic management and outcomes of suspected pulmonary embolism. Ann Intern Med. 2006;144:157-164.

14. Kline JA, Hernandez-Nino J, Jones AE, et al. Prospective study of the clinical features and outcomes of emergency department patients with delayed diagnosis of pulmonary embolism. Acad Emerg Med. 2007;14:592-598. doi: 10.1197/j. aem.2007.03.1356.

15. Guo DJ, Zhao C, Zou YD, et al. Values of the Wells and revised Geneva scores combined with D-dimer in diagnosing elderly pulmonary embolism patients. Chin Med J (Engl). 2015;128:1052-1057. doi: 10.4103/0366-6999.155085.

16. Di Marca S, Cilia C, Campagna A, et al. Comparison of Wells and Revised Geneva Rule to Assess Pretest Probability of
Pulmonary Embolism in High-Risk Hospitalized Elderly Adults. J Am Geriatr Soc. 2015;63:1091-1097. doi: 10.1111/ jgs.13459.

17. Buchanan I, Teeples T, Carlson M, et al. Pulmonary Embolism Testing Among Emergency Department Patients Who Are Pulmonary Embolism Rule-out Criteria Negative. Acad Emerg Med. 2017. doi: 10.1111/acem.13270. [Epub ahead of print]

18. de la Mora Cervantes R, Dennie C. Triple rule-out cardiac computed tomography: is it finally a reality? Minerva Cardioangiol. 2017;65:225-234. doi: 10.23736/S00264725.16.04272-9.

19. Dobra M, Bordi L, Nyulas T, et al. Computed Tomography - an Emerging Tool for Triple Rule-Out in the Emergency Department. A Review. Journal of Cardiovascular Emergencies. 2017;3:36-40. doi: 10.1515/jce-2017-0005.

20. Sawyer KN, Shah P, Qu L, et al. Triple Rule Out versus CT Angiogram Plus Stress Test for Evaluation of Chest Pain in the Emergency Department. West J Emerg Med. 2015;16:677-682. doi: 10.5811/westjem.2015.6.25958.

21. Chae MK, Kim EK, Jung KY, et al. Triple rule-out computed tomography for risk stratification of patients with acute chest pain. J Cardiovasc Comput Tomogr. 2016;10:291-300. doi: 10.1016/j.jcct.2016.06.002.

22. Le Gal G, Righini M, Roy PM, Sanchez O, Aujesky D, Bounameaux H, Perrier A. Prediction of pulmonary embolism in the emergency department: the revised Geneva score. Ann Intern Med. 2006;144:165-171.

23. Woolf SH, Grol R, Hutchinson A, Eccles M, Grimshaw J. Clinical guidelines: Potential benefits, limitations, and harms of clinical guidelines. BMJ. 1999;318:527-530.

24. Wicki J, Perneger T, Junod A, et al. Assessing clinical probability of the pulmonary embolism in the emergency ward: a simple score. Arch Intern Med. 2001;161:92-97.

25. Douma RA, Mos IC, Erkens PM, et al. Performance of 4 clinical decision rules in the diagnostic management of acute pulmonary embolism. A prospective cohort study. Ann Intern Med. 2011;154:709-718. doi: 10.7326/0003-4819-154-11201106070-00002.

26. Wells PS, Anderson DR, Rodger M, et al. Derivation of a simple clinical model to categorize patients probability of pulmonary embolism: increasing the models utility with the SimpliRED D-dimer. Thromb Haemost. 2000;83:416-420.

27. El Wahsh RA, Agha MA. Clinical probability of pulmonary embolism: Comparison of different scoring systems. In Egyptian Journal of Chest Diseases and Tuberculosis. 2012; 61:419-424. doi: 10.1016/j.ejcdt.2012.07.002.

28. Miniati M, Bottai M, Monti S. Simple and accurate prediction of the clinical probability of pulmonary embolism. Am J Respir Crit Care Med. 2008;178:290-294. doi: 10.1164/rccm.2008022070C.

29. Lucassen W, Geersing G, Erkens PM, et al. Clinical Decision $\underline{\text { Rules for }}$ Excluding Pulmonary Embolism: A Meta-analysis. Ann Intern Med. 2011;155:448-460. doi: 10.7326/0003-4819155-7-201110040-00007.

30. Aujesky D, Roy PM, Le Manach CP, et al. Validation of a model to predict adverse outcomes in patients with pulmonary embolism. Eur Heart J. 2006;27:476-481.

31. Spirk D, Aujesky D, Husmann M, et al. Cardiac troponin testing and the simplified Pulmonary Embolism Severity Index. The SWIss Venous Thrombo Embolism Registry (SWIVTER). Thromb Haemost. 2011;106:978-984. doi: 10.1160/TH11-060371. 
32. Prandoni $\mathrm{P}$, Lensing AWA, Prins $\mathrm{MH}$, et al. Prevalence of Pulmonary Embolism among Patients Hospitalized for Syncope. N Engl J Med. 2016;375:1524-1531. doi: 10.1056/ NEJMoa1602172.

33. Oqab Z, Ganshorn H, Sheldon R. Prevalence of pulmonary embolism in patients presenting with syncope. A systematic review and meta-analysis. Am J Emerg Med. 2017;pii:S0735-6757(17)30740-4. doi: 10.1016/j. ajem.2017.09.015. [Epub ahead of print]

34. Frizell A, Fogel N, Steenblik J, et al. Prevalence of pulmonary embolism in patients presenting to the emergency department with syncope. Am J Emerg Med. 2017;pii:S0735-6757(17)30639-3. doi: 10.1016/j. ajem.2017.07.090. [Epub ahead of print]

35. TripodiA, ChantarangkulV, ClericiM, NegriB, GalliM, Mannucci PM. Laboratory control of oral anticoagulant treatment by the INR system in patients with the antiphospholipid syndrome and lupus anticoagulant. Results of a collaborative study involving nine commercial thromboplastins. Br J Haematol. 2001;115:672-678.

36. Asherson RA, Piette JC. The catastrophic antiphospholipid syndrome: acute multiorgan failure associated with antiphospholipid antibodies: a review of 31 patients. Lupus. 1996;5:414-417.

37. Miyakis S, Lockshin MD, Atsumi T, et al. International consensus statement on an update of the classification criteria for definite antiphospholipid syndrome (APS). J. Thromb Haemost. 2006;4:295-306. doi: 10.1111/j.15387836.2006.01753.x.

38. Gorki H, Malinovski V, Stanbridge RDL. The antiphospholipid syndrome and heart valve surgery. Eur J CardioThorac Surg. 2008;33:168-181. doi: 10.1016/j.ejcts.2007.11.004.

39. Cervera R, Serrano R, Pons-Estel GJ, et al. Morbidity and mortality in the antiphospholipid syndrome during a 5-year period: a multicenter prospective study of 1,000 patients. Ann Rheum Dis. 2015;74:1011-1018. doi: 10.1136/ annrheumdis-2013-204838.

40. Negrini S, Pappalardo F, Murdaca G, Indiveri F, Puppo F. The antiphospholipid syndrome: from pathophysiology to treatment. Clin Exp Med. 2017;17:257-267. doi: 10.1007/ s10238-016-0430-5.

41. Girardi G, Redecha P, Salmon JE. Heparin prevents antiphospholipid antibody-induced fetal loss by inhibiting complement activation. Nat Med. 2004;10:1222-1226.

42. Gex G, Gerstel E, Righini M, et al. Is atrial fibrillation associated with pulmonary embolism? J Thromb Haemost. 2012;10:347351.

43. Bikdeli B, Abou Ziki MD, Lip GY. Pulmonary Embolism and Atrial Fibrillation: Two Sides of the Same Coin? A Systematic Review. Semin Thromb Hemost. 2017. doi: 10.1055/s-00361598005. [Epub ahead of print] 